\title{
Cardiac tamponade as the initial manifestation of severe hypothyroidism: A case report
}

\author{
Ronny Cohen $^{1,2^{*}}$, Pablo Loarte ${ }^{2,3}$, Simona Opris ${ }^{2}$, Brooks Mirrer ${ }^{1,2}$ \\ ${ }^{1}$ NYU School of Medicine, New York, USA \\ ${ }^{2}$ Division of Cardiology, Woodhull Medical Center, New York, USA \\ ${ }^{3}$ Division of Nephrology and Hypertension, Brookdale University Hospital and Medical Center, New York, USA \\ Email: ronny.cohen@nychhc.org
}

Received 11 May 2012; revised 14 June 2012; accepted 23 June 2012

\begin{abstract}
Background: Hypothyroidism is a commonly seen condition. The presence of pericardial effusion with cardiac tamponade as initial manifestation of this endocrinological condition is very unusual. Objectives: In hypothyroidism pericardial fluid accumulates slowly, allowing adaptation and stretching of the pericardial sac, sometimes accommodating a large volume. Case Report: A 39 year-old female presented with chest pain, dyspnea and lower extremity edema for 1 day. Bradycardia, muffled heart sounds and severe hyper- tension were noticed. Chest radiograph showed an enlarged cardiac silhouette. A bedside echocardiogram revealed a cardiac tamponade, later she developed sudden hypotension and bradycardia that resolved after pericardiocentesis of 1 liter of pericardial fluid. The further laboratory evaluation revealed a TSH value of $69.3 \mathrm{miU} / \mathrm{L}$ and low $\mathrm{T} 3$ and free T4. The patient later developed re-accumulation of pericardial fluid with the need for creation of pericardial window. Conclusion: When the classic Beck's triad is not present and bradycardia accompanies a cardiac tamponade, hypothyroidism should be strongly suspected. The requirement for thyroid hormone supplement is critical and is well reported. There is a chance of recurrence even after starting levothyroxine supplementation; and the associated hypertension usually requires treatment with more than one drug.
\end{abstract}

Keywords: Cardiology; Cardiac Tamponade; Pericardial Effusion; Hypothyroidism; Emergency Department; Pericardiocentesis; Thyroid

\section{INTRODUCTION}

Pericardial effusion due to hypothyroidism was reported as early as 1918 and subsequently in 1925 [1]. The total

*Corresponding author. incidence of pericardial effusion secondary to hypothyroidism varies in different studies from $30 \%$ to $80 \%$ [2]. Cardiac tamponade as a complication of hypothyroidism is very rare [3]. Until 1992, there were less than 30 cases described and even more recently there are only few cases found in the world literature. This low incidence is most likely due to slow accumulation of fluid and gradual pericardial distention [4]. Hypothyroidism is characterized by low metabolic demands and therefore, despite a depressed cardiac contractility and cardiac output, cardiac function remains sufficient to sustain the workload imposed on the heart. Another feature that distinguishes cardiac tamponade caused by hypothyroidism from the other causes of tamponade is the absence of sinus tachycardia as a mechanism to maintain cardiac output [5]. We report a case of cardiac tamponade as a presenting manifestation of hypothyroidism.

\section{CASE PRESENTATION}

A 39 year-old homeless female presented to the emergency department (ED) because of chest pain for 1 day, bilateral leg pain, shortness of breath, decreased exercise tolerance and swollen lower extremities. Her past history included uncontrolled diabetes mellitus type 2 and hypertension. Vital signs on admission were blood pressure 202/117 mmHg, heart rate $75 \mathrm{bpm}$, respiratory rate 16 breaths/minute and temperature $98.1^{\circ} \mathrm{F}$. Physical examination showed an obese, confused patient, puffy eyes and face, thyroid gland was non-tender, no nodules palpated. Cardiac sounds were distant, and patient had bilateral lower extremity pitting edema; and delayed deep tendon reflexes. Pulses were present in all four extremities, but weak.

Laboratory analysis done in ED revealed: Hb 11.7 g/dL, glucose $219 \mathrm{mg} / \mathrm{dL}$, CK $134 \mathrm{U} / \mathrm{L}$, troponin I 0.036 $\mathrm{ng} / \mathrm{mL}$, (normal $<0.05 \mathrm{ng} / \mathrm{mL}$ ) BNP $42 \mathrm{pg} / \mathrm{mL}$, arterial blood gases $\left(\mathrm{FiO}_{2} 21 \%\right)$ : $\mathrm{pH}$ 7.41, $\mathrm{PaCO}_{2} 52 \mathrm{mmHg}, \mathrm{PaO}_{2}$ $71 \mathrm{mmHg}$, Sat $\mathrm{O}_{2} 94 \%$. Chest radiograph showed massive enlargement of cardiac silhouette (Figure 1). The ECG with sinus rhythm, first degree AV block, right axis 


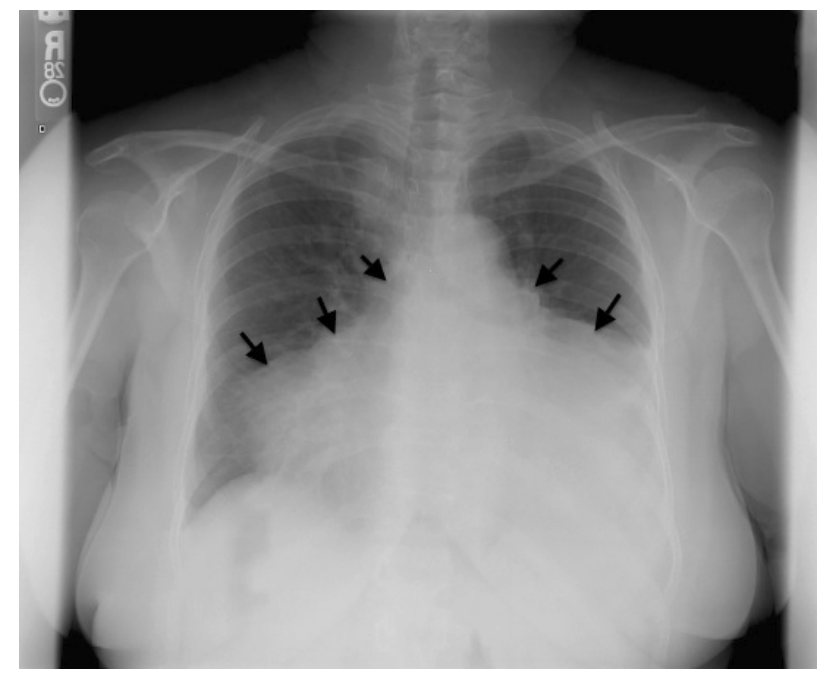

Figure 1. Postero-anterior chest radiography shows a massive bottle-shaped heart (arrows) typical of cardiac tamponade.

deviation, borderline low voltage in frontal leads, late precordial RS transition and nonspecific abnormal $\mathrm{T}$ waves in II III aVF V1 V3 (Figure 2). A bedside echocardiogram was done, showing large pericardial effusion, Right Atrium (RA) and Right Ventricle (RV) diastolic collapse and respirophasic, transmitral flow variation suggesting tamponade (Figures 3 and 4). After a few minutes she became drowsy, hypotensive and bradycardic, with heart rate of $36 \mathrm{bpm}$. She was immediately intubated for airway protection and to decrease the work of breathing.

Blind sub-xiphoid pericardocentesis was performed and $100 \mathrm{cc}$ of sero-sanguinous fluid was aspirated which resulted in immediate improvement in blood pressure $(102 / 54 \mathrm{~mm} / \mathrm{Hg})$ and HR (56 - $60 \mathrm{bpm})$. Additionally the patient received aggressive fluid resuscitation. The thoracic surgeon repeated the needle pericardiocentesis and a total of 1 liter of pericardial fluid were drained with the same characteristics as initally. Blood pressure after the end of pericardiocentesis remained elevated with a value of 163/92 mmHg.

The medical course included 5 days of ICU admission. Subsequent electrocardiograms and cardiac biomarkers where negative for acute myocardial infarction. On the second day of admission, the echocardiogram was repeated after removal of pericardial catheter and showed a large pericardial effusion all around the heart with diastolic collapse of RV, RA consistent with early recurrence of tamponade, normal LV systolic function with an ejection fraction of $65 \%$. Surgery performed a pericardial window for re-accumulation fluid and a Jack-Pratt drain was left in place until patient was discharged, draining a total of $1 \mathrm{~L}$ of additional fluid over 2 days. Pericardial fluid analysis showed glucose $171 \mathrm{mg} / \mathrm{dL}$, protein $6.2 \mathrm{~g} / \mathrm{dL}$, cell count RBCs 125,500 /cu mm, WBCs 4750/cu mm: Neutrophils $70 \%$, Lymphocytes $28 \%$, Mono $2 \%$. There were no malig

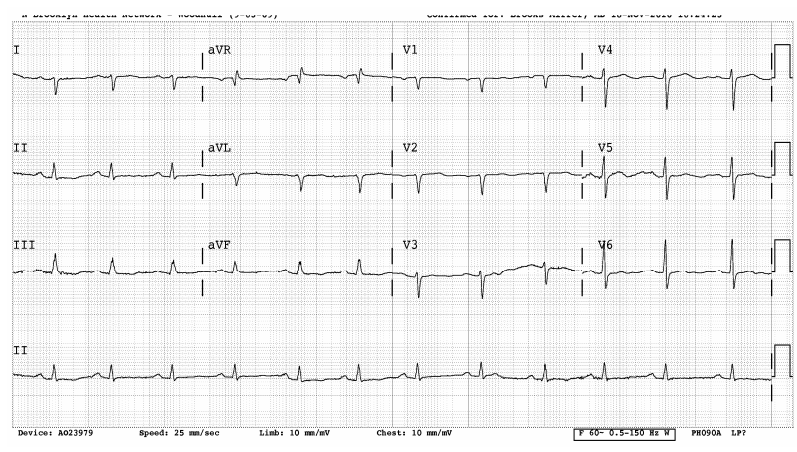

Figure 2. ECG at ED presentation with some QRS variation.

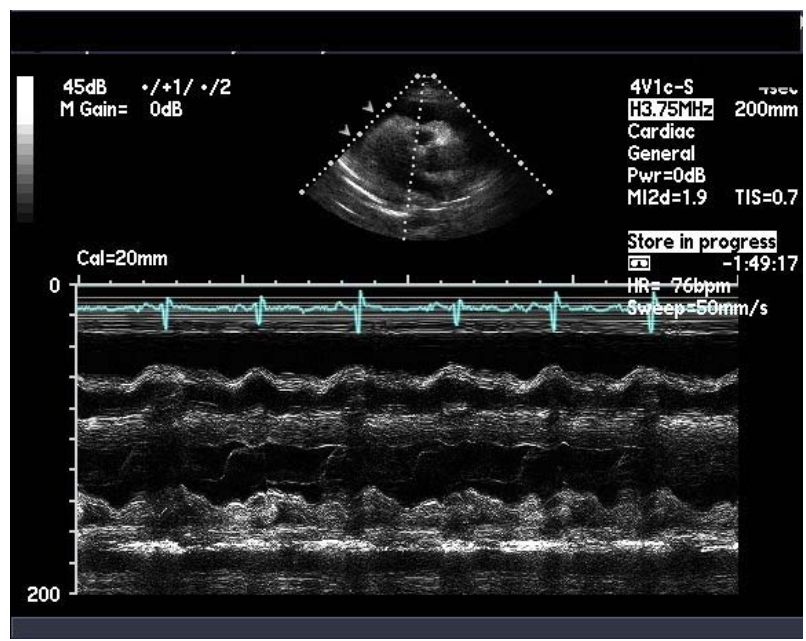

Figure 3. Above: Echocardiogram showing diastolic collapse; Below: Echocardiogram M mode, mitral window level showing variation in RV and LV volumes suggesting tamponade. $\mathrm{RV}$ varies in compression during diastole.

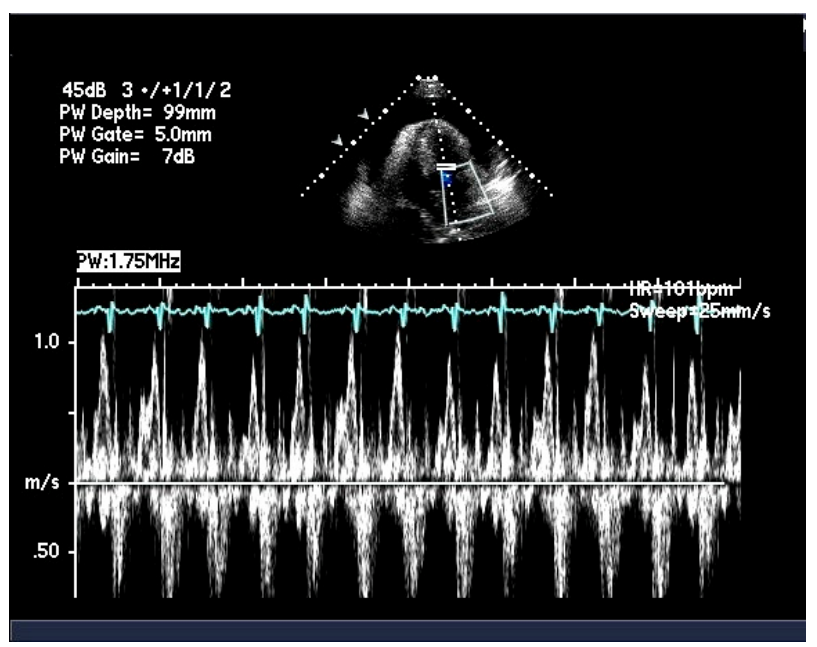

Figure 4. Respirophasic variation in magnitude of E-point velocity of diastolic trans-mitral flow, greater than $\mathbf{5 0 \%}$ suggesting tamponade

nant cells, pericardial cultures were negative (including Mycobacterium tuberculosis), PCR was negative, ANA 
was negative, PPD was negative, serum LDH 340 U/L, CRP $2.3 \mathrm{mg} / \mathrm{mL}$. Postoperatively patient had low tidal volumes, poor inspiratory effort and difficulties weaning from mechanical ventilation due to episodes of apnea and remained on mechanical ventilation for a total of 3 days. Thyroid function tests revealed a TSH of 69.13 ug/dL, T4 of $3.0 \mathrm{ug} / \mathrm{dL}$, T3U of $38.5 \mathrm{ng} / \mathrm{dL}$, and free T4 of $0.4 \mathrm{ng} / \mathrm{dL}$. Diagnosis of hypothyroidism was made and cardiac tamponade secondary to hypothyroidism was established as the cause.

Even though our patient had significant echocardiographic evidence of early cardiac tamponade, she remained with an elevated blood pressure and required the use of beta-blockers, ACE inhibitors and diuretics to control the blood pressure. The patient was started on levothyroxine $75 \mathrm{mcg}$ daily and later transferred to medicine floor and discharged with a regimen of levothyroxine 75 mcg and instruction for outpatient follow-up and reassessment of the TSH level. Secondary causes of hypertension were excluded during the hospitalization. The patient was followed in the outpatient clinic and was not compliant with the treatment regimen.

\section{DISCUSSION}

Cardiac tamponade rarely occurs with severe hypothyroidism and myxedema [5]. Our case is particularly unique due to the fact that presenting symptoms were not typical and the patient was severely hypertensive.

\subsection{Pathophysiology of Cardiac Tamponade in Hypothyroidism}

The normal pericardium is a sac composed of 2 layers: An outer parietal and an inner visceral pericardium. Between these 2 layers, there is a space filled by serous fluid which amount is no more than 15 to $30 \mathrm{~mL}$ [6]. The visceral is composed of mesothelial cells and the parietal is formed of collagen fibers and a small amount of elastin [7]. The pericardium completely surrounds the heart and attaches to the adjacent structures and vessels. It is well innervated and secretes substance as prostaglandins that play a role in the cardiac function. The pericardium limits acute cardiac dilation and enhances the mechanical interaction of the cardiac chambers as a result of its relatively inelastic properties.

In response to long-standing stress, the pericardium dilates, shifting the pericardial pressure-volume relation substantially to the right [7]. When there is slow accumulation of fluid, the parietal pericardium compliance increases. The increasing amounts of fluid increase at the same time the intrapericardial pressure, while the central venous pressure responds by increasing to maintain a gradient that allows cardiac filling. When the pericardial compliance reaches its maximal capacity, the intrapericardial pressure equalizes with the right ventricular diastolic pressure and later with the left. At this point, the cardiac output drops and the circulation is maintained by an increase in heart rate, contractility and peripheral vasoconstriction [6]. If the fluid accumulates slowly, large amounts can occupy the space but in case of rapid fluid accumulation, the compliance capacity is exceeded. The amount of fluid in the pericardial effusions associated with hypothyroidism can be as large as 5 - 6 L [8].

The mechanism by which effusion and subsequently tamponade occur is part of a generalized polyseropathy [4]. Inadequate lymphatic drainage may be the mechanism by which the exudates are produced in the serous cavities of patient with hypothyroidism [9].

\subsection{Cardiac Tamponade}

The primary phenomenon in tamponade is a compression of all cardiac chambers after the pericardial content reaches the limit of pericardial reserve volume. With smaller cardiac chambers, the myocardial diastolic compliance is reduced and cardiac inflow becomes limited, ultimately equalizing mean diastolic pericardial and chamber pressures. This equalization of pressures is the hallmark of cardiac tamponade [10]. Etiology of cardiac tamponade includes idiopathic pericarditis, iatrogenic effusion, infection, malignancy; metabolic factors, collagen vascular disease, tuberculosis, traumatic, and drug induced acute hemorrhage and post-cardiac surgery. If the early presentation of tamponade is with bradycardia, the differential diagnosis can be narrowed to conditions such as uremia, SLE pericarditis, Infectious agents such as coxiella and viral myocarditis [6].

Clinical features depend on the rapidity of accumulation of fluid in the pericardial space and include chest pain and dizziness. Tachycardia is the rule and is part of the main findings. Bradycardia can be present in uremic and hypothyroid patients. Venous pressure is elevated and manifested as jugular venous distention. Pulsus paradoxus is also present but may be absent if severe hypotension is present. Classic findings include Beck's triad (Raised venous pressure, arterial hypotension and distant heart sounds) $[7,10]$.

\subsection{Clinical Manifestations of Hypothyroidism on Cardiovascular System}

The clinical presentation of overt hypothyroidism may not be obvious and most patients have few symptoms and signs. Bradycardia and systemic hypertension, with narrow pulse pressure and slightly increased mean arterial pressure, and some degree of exercise impairment are the most common findings in patients with overt hypothyroidism. Systemic hypertension in overt hypothy- 
roidism is likely due to the remarkable increase in peripheral vascular resistance and increase in arterial stiffness, likely resulting from myxedema of the arterial wall. [11].

\subsection{Diagnosis of Hypothyroidism-Induced Cardiac Tamponade}

In hypothyroidism there is an increased capillary permeability and impaired lymphatic drainage with protein leakage into the interstitial space, resulting in pericardial effusion. Furthermore there is increased salt and water retention [4,8]. As mentioned previously, Beck's triad can appear clinically, but in our case the patient only had muffled heart sounds. The presence of hypertension in our patient can be explained by a possible excessive adrenergic drive caused by the tamponade. A hypothyroid status can be associated with diastolic hypertension but in patients with hypertensive hypothyroid massive effusion, the hypertensive status can persist following pericardiocentesis and treatment of hypothyroidism [5]. The initial blood pressure in the case was 202/117 mmHg and after pericardiocentesis, the blood pressure decreased only to $180 / 104 \mathrm{mmHg}$ and required therapy, with three hypertensive medications. The use of thyroid replacement therapy did not contribute to the control of the hypertension. Another suggested explanation to this is the possible presence of aortic stiffness [12].

Bradycardia in hypothyroid cardiac tamponade has been evident in several studies and is suggesting to be related to a decreased sympathetic activity, but indirect measurements of sympathetic activity showed that is elevated. There are also some evidence of blunted sympathetic excitatory and tachycardic response to hypotension and depressed arterial baroreflex with elevated dependence on the resting sympathetic tone that can explain the presence of bradycardia [13].

The ECG findings are very well known and described. Decreased voltage with electrical alternants. It is important to mention that our patient presented $\mathrm{T}$ wave flattening which has been mentioned to be associated with myxedematous heart disease [14]. QT prolongation has been also observed in patients with hypothyroidism and tamponade but it was not present in our patient. The QT prolongation is most likely secondary to the hypothyroidism that tamponade. Presences of sinus bradycardia, low QRS voltage, diffuse flat $\mathrm{T}$ waves and Long QT have a positive predictive value for hypothyroidism [15].

Echocardiogram in $\mathrm{M}$ or 2-D mode can be performed showing diastolic collapse of right atrium, right ventricle and left atrium. Inferior vena cava can be dilated but this finding was not present in our case. Left ventricule can show "pseudohypertrophy" due to increased diastolic wall thickness; in our case there was presence of hyper- throphy. However, this can be explained by two interrelated conditions; hypertension and hypothyroidism. The hypothyroidism produces arterial wall stiffness and induces hypertension and hypertension by itself can produce LV concentric hypertrophy if it is not treated [11, 16].

The fluid characteristic in hypothyroid cardiac tamponade is typically an effusion, with a color that goes from clear to golden hue, with predominance of lymphocytes and high protein content. In our case, the fluid obtained in two drainage collections was sero-sangineous and we consider trauma as a possible cause. Other superimposed causes like infection, connective tissue disease and trauma were ruled out, but the possibility of spontaneous pericardial hemorrhage also can exist $[8,14,17]$.

\section{TREATMENT}

Treatment depends on the hemodynamic state of the patient. Removal of even a small amount of pericardial fluid (about $50 \mathrm{ml}$ ) produces considerable hemodynamic and symptomatic improvement because of the steep pericardial pressure volume relationship. Early tamponade with only mild hemodynamic compromise may be treated conservatively with close observation, fluid restriction and therapy aimed at underlying cause. A majority of the cases will require surgical drainage procedure. Pericardiocentesis followed by a surgical drainage with a pericardial window if effusion recurs is a reasonable approach [18]. In our case the fluid re-accumulated twice within a few hours, but can happen in days or a month $[4,19,20]$. Thus, in pericardial effusion and tamponade due to hypothyroidism, recurrence of effusion after needle drainage of pericardial fluid occurs often and should be anticipated. Therapeutic measures that reduce venous filling pressures or effective cardiac output should be avoided [21]. Echo-guided pericardiocentesis is well tolerated by patients, is a quick procedure and can be performed even in unstable patients. The success rate is about $97 \%$ and the preferred method of approach is the subxiphoid region [22]. There is generally a residue of effusion that disappears following appropriate therapy over a period of 1 month to 1 year and for this reason treatment with increasing doses of Levothyroxine is imperative. Thyroid replacement therapy is necessary over a long period of time, requiring close follow-up [4].

\section{CONCLUSION}

Hypothyroidism with cardiac tamponade as a presenting symptom is a rare condition. Cardiac tamponade is a lifethreatening condition. Physicians should consider hypothyroidism as a differential diagnosis in patients with pericardial effusions, especially when progressing to cardiac tamponade. A high index of suspicion must be main- 
tained for timely diagnosis of cardiac tamponade due to severe hypothyroidism followed by prompt intervention because this is a treatable cause of cardiogenic shock that can be fatal if unrecognized. Pericardiocentesis is necessary only when cardiac tamponade develops or when large amounts of effusions are identified. Mild pericardial effusion and early tamponade does respond to levothyroxine supplementation over a long period of time, necessitating long term follow-up.

\section{ACKNOWLEDGEMENTS}

Jay Jacobs for echo illustration. Carla Mayers and Gail Charles for excellent typing. This is an original manuscript of original research and discussion presented for review and possible publication for advancement of medical education. It has not been submitted to any other journal. There are no financial interests tied to this original research, neither is there any other side support. The authors listed originally prepared all materials. The authors listed have contributed to, read and approved the manuscript. The authors listed have no conflicts of interest.

\section{REFERENCES}

[1] Rernam, V.J., Chichgar, J.A., Patkar, L.A., et al. (1983) Myxedema and pericardial effusion with cardiac tamponade (a case report). Journal of Postgraduate Medicine, 29, 188-190.

[2] Arthur, S., Beeharry-Panray, G., Fitzgerald, J., et al. (2009) Hypothyroidism presenting with recurrent pericardial tamponade. BMJ Case Reports.

[3] Handler, J. (2007) Hypertensive emergency with cardiac tamponade associated with hypothyroidism. The Journal of Clinical Hypertension, 9, 67-72. doi:10.1111/j.1524-6175.2007.06343.x

[4] Jimenez-Nacher, J.J., de Alonso, N., Vega, B., et al. (1993) Cardiac tamponade as a presentation of primary hypothyroidism in a young woman. Revista Clínica Española, 193, 290-292.

[5] Wang, J.L., Hsieh, M.J., Lee, C.H., et al. (2010) Hypothyroid cardiac tamponade: Clinical features, electrocardiography, pericardial fluid and management. The American Journal of the Medical Sciences, 340, 276-281. doi:10.1097/MAJ.0b013e3181e664c6

[6] Roy, C.L., Minor, M.A., Brookhart, M.A., et al. (2007) Does this patient with a pericardial effusion have a cardiac tamponade? JAMA, 297, 1810-1818. doi:10.1001/jama.297.16.1810

[7] Little, W.C. and Freeman, G.L. (2006) Pericardial disease. Circulation, 113, 1622-1632. doi:10.1161/CIRCULATIONAHA.105.561514

[8] Goswami, R., Tandon, N., Singh, B., et al. (1996) Circulatory collapse in a 30-year-old amenorrheic woman. Postgraduate Medical Journal, 72, 501-509. doi:10.1136/pgmj.72.850.501

[9] Parving, A., Ostri, B., Bretlau, P., et al. (1979) Mechanisms of edema formation in myxedemaincreased protein extravasation and relatively slow lymphatic drainage. The New England Journal of Medicine, 301, 460-465. doi:10.1056/NEJM197908303010902

[10] Spodick, D.H. (2003) Acute cardiac tamponade. The New England Journal of Medicine, 349, 684-690. doi:10.1056/NEJMra022643

[11] Fazio, S., Palmieri, E.A., Lombardi, G. et al. (2004) Effects of thyroid hormone on cardiovascular system. Recent Progress in Hormone Research, 59, 31-50. doi:10.1210/rp.59.1.31

[12] Dernellis, J. and Panaretou, M. (2002) Effects of thyroid replacement therapy on arterial blood pressure in patients with hypertension and hypothyroidism. American Heart Journal, 143, 718-724. doi:10.1067/mhj.2002.120766

[13] Foley, C.M., McAllister, R.M. and Hasser, E.M. (2001) Thyroid status influences baroreflex function and autonomic contributions to arterial pressure and heart rate. American Journal of Physiology-Heart and Circulatory Physiology, 280, H2061-H2068.

[14] Wang, J.L., Hsieh, M.J., Lee, C.H., et al. (2010) Hypothyroid cardiac tamponade: Clinical features, electrocardiography, pericardial fluid and management. The American Journal of the Medical Sciences, 340, 276-281. doi:10.1097/MAJ.0b013e3181e664c6

[15] Glancy, D.L. and Wang, W. (2007) Electrocardiogram in a 55-year-old woman with an endocrinedisorder. Baylor University Medical Center Proceedings, 20, 81-82.

[16] Maisch, B., Seferovic, P.M., Ristic, A.D., et al. (2004) Guidelines for the diagnosis and management of pericardial diseases. Executive summary. The task force on the diagnosis and management of pericardial diseases of the euro- pean society of cardiology. European Heart Journal, 25, 587-610.

[17] Hurtado, J.J., Guevara, W., Ramos, E., et al. (2011) Hypothyroidism in a five-year-old boy with rhabdomyolysis and recent history of cardiac tamponade: A case report. Journal of Medical Case Reports, 5, 515-519. doi:10.1186/1752-1947-5-515

[18] Imazio, M., Spodick, D.H., Brucato, A., et al. (2010) Controversial issues in the management of pericardial diseases. Circulation, 121, 916-928. doi:10.1161/CIRCULATIONAHA.108.844753

[19] Karu, A.K., Khalife, W.I. and Houser, R. (2005) Impending cardiac tamponade as a primary presentation of hypothyroidism: Case report and review of literature. Endocrine Practice, 11, 265-271.

[20] Vasquez, A. (1993) Cardiac tamponade and hypothyroidism. Intensive Care Medicine, 19, 241. doi:10.1007/BF01694781

[21] Alsever, R.N., Stjernholm, et al. (1975) Cardiac tamponade in myxedema. The American Journal of the Medical Sciences, 269, 117-121. doi:10.1097/00000441-197501000-00012

[22] Gumrukcuoglu, H.A., Odabasi, D. and Akdag, S. (2011) Management of cardiac tamponade: A comparative study between echo-guided pericardiocentesis and surgery-A report of 100 patients. Cardiology Reasearch and Practice, 38, $7 \mathrm{p}$. 